\title{
Time-dependent double-diffusive convection due to salt-stratified fluid layer with differential heating in an inclined cavity
}

\author{
YAU-MING CHEN and JIUN-KAE LIOU \\ Department of Mechanical Engineering, National Taiwan University, Taipei, Taiwan 10764, \\ Republic of China
}

(Received 21 July 1995)

\begin{abstract}
This study investigates the time-dependent double-diffusive convection of $\mathrm{NaCl}-\mathrm{H}_{2} \mathrm{O}$ concerning the stably stratified salt fluid layer with lateral heating in an inclined cavity. The $N-S$ equations are solved via a finite difference method with $R a=1 \times 10^{7}$ and $R a_{\mathrm{s}}=5 \times 10^{7}$. The plots of stream function, isotherms and isosolutal lines at inclined angles from $\gamma=75^{\circ}$ to $-75^{\circ}$ during a long period of time are presented and systematically discussed. At early time, there are two types of instability onset. At $\gamma<75^{\circ}$, the cells rotate strikingly in one sense of rotation, but weakly in the other, while at $\gamma \geqslant 75^{\circ}$ the comparable vortexes rotating in a different sense are generated. The onset of instability is nonsymmetric, being faster at negative angles than at positive ones. The multi-layered structure is formed with a sharp solutal difference and a wavy distribution of temperature at all inclined angles. The distribution of local Nusselt number is periodic due to the layered structure. The average Nusselt number with respect to time reveals more detailed information about the heat transfer behavior of layer forming, propagating and merging. The prediction of final layer thickness and flow field agrees well with the experiments and flow visualization. Copyright (C) 1996 Elsevier Science Ltd.
\end{abstract}

\section{INTRODUCTION}

Double-diffusive convection due to a salt stratified fluid layer with differential heating has received increasing research attention. In nature, it extensively exists in oceanography, geophysics and geology [1-3]. There are many important applications in engineering, such as for energy storage systems, solar engineering and some material processings [1, 2].

The solutal effect is stable, but the thermal effect would be unstable. The most interesting feature is that it will generate a layered structure with sharp interfaces. Within the layer, the solute distribution is uniform, but the temperature distribution may exhibit two different types: wavy or uniform in the vertical direction. The relevant flow types are horizontal cellular motion and well-mixed fluid layers [3]. The decisive reasons are magnitude and orientation of thermal and solutal gradients.

For lateral heating, Thrope et al. [4] first clarified the secondary flow pattern of a layered structure that resulted from the onset of instability in a narrow vertical slot. Chen et al. [5] carried out experiments in a tank with natural length scale $\eta=\alpha \Delta T / \phi_{0}$, defining the modified Rayleigh number $R a_{\eta}$, and proposed the critical value $R a_{\eta, \mathrm{cr}} \cong 15000 \pm 2500$ such that the simultaneous cells are generated on the sidewall if $R a_{\eta}$ is larger than the critical value; while some successive cells only are found in the vicinity of the top and bottom walls if $R a_{\eta}$ is smaller than the critical value. The final layer thickness $h$ is estimated to be about
(0.6-0.9) $\eta$. Huppert and Turner [6] and Huppert et al. [7] measured the final layer thickness in a wide range, and found that $h=0.62 \pm 0.05 \eta$ as $R a_{\eta}>10^{5}$, which is independent of the modified Rayleigh number. More detailed discussions about the cell propagation and layered structure can be found in the studies by Tanny and Tsinober [8] and Jeevaraj and Imberger [9].

Recently, Lee and Hyun [10], Kamakura and Ozoe [11] and Dosch and Beer [12] numerically simulated the problem by finite difference $[10,12]$ or finite element [11] method. They reported the flow field, heat and mass transfer characteristics in the closed rectangular [10], opened rectangular [11] and the concentric cavities [12]. Experimental results were also presented by using the shadowgraph [11] and holographic interferometry methods [12]. All of them showed their numerical predictions were similar to their experimental results or the data in literatures.

Unlike the lateral heating when the heat flux is imposed from the bottom, the vectors of two gradients are parallel and opposed to each other such that the interaction is intensive, and other types of instability occur in the flow. A row of vortexes rotating with different sense is generated at the bottom, as shown in the theoretical analysis by Chen [13] and the numerical simulation by Kazmierczak and Poulikakos [14]. The vortexes form the plume structure, eventually one or more well-mixed fluid layer generates in the flow, which can be seen in the numerical results of Kazmierczak and Poulikakos [14] and the exper- 


\section{NOMENCLATURE}

\begin{tabular}{|c|c|}
\hline$A r$ & aspect ratio, $H / L$ \\
\hline$C$ & dimensional solute $[\mathrm{wt} \%]$ \\
\hline$C_{\mathrm{h}}$ & $\begin{array}{l}\text { dimensional maximum solute at initial } \\
{[w t \%]}\end{array}$ \\
\hline$C_{1}$ & $\begin{array}{l}\text { dimensional minimum solute at initial } \\
{[w \mathrm{t} \%]}\end{array}$ \\
\hline$c$ & $\begin{array}{l}\text { nondimensional solute, } \\
\left(C-C_{1}\right) /\left(C_{\mathrm{h}}-C_{1}\right)\end{array}$ \\
\hline$D$ & mass diffusivity $\left[\mathrm{m}^{2} \mathrm{~s}^{-1}\right]$ \\
\hline$g$ & gravitational acceleration $\left[\mathrm{m} \mathrm{s}^{-2}\right]$ \\
\hline$H$ & height of cavity [m] \\
\hline$h$ & average size of layer thickness [m] \\
\hline$L$ & width of cavity [m] \\
\hline Le & Lewis number, $\alpha / D$ \\
\hline$N u$ & local Nusselt number, equation (12) \\
\hline$\overline{N u}$ & $\begin{array}{l}\text { average Nusselt number, equation } \\
\text { (12) }\end{array}$ \\
\hline$P$ & dimensional pressure $\left[\mathrm{N} \mathrm{m}^{-2}\right]$ \\
\hline$p$ & nondimensional pressure, $p L^{2} /\left(\rho \alpha^{2}\right)$ \\
\hline $\operatorname{Pr}$ & Prandtl number, $v / \alpha$ \\
\hline$R a$ & $\begin{array}{l}\text { thermal Rayleigh number, } \\
g \beta_{\mathrm{T}} \Delta T L^{3} /(\alpha \nu)\end{array}$ \\
\hline$R a_{\mathrm{s}}$ & $\begin{array}{l}\text { solutal Rayleigh number, } \\
\mathrm{g} \beta_{\mathrm{s}} \Delta C L^{3} /(\alpha v)\end{array}$ \\
\hline$R_{\rho}$ & buoyancy ratio, $\beta_{\mathrm{s}} \Delta C / \beta_{\mathrm{T}} \Delta T$ \\
\hline$R a_{\eta}$ & $\begin{array}{l}\text { modified Rayleigh number, } \\
g \beta_{\mathrm{T}} \Delta T \eta^{3} /(\alpha \nu)\end{array}$ \\
\hline$R a_{\eta \mathrm{cr}}$ & $\begin{array}{l}\text { critical value of modified Rayleigh } \\
\text { number }\end{array}$ \\
\hline$T$ & dimensional temperature $[\mathrm{K}]$ \\
\hline$T_{\mathrm{h}}$ & dimensional highest temperature $[\mathrm{K}]$ \\
\hline$T_{1}$ & dimensional lowest temperature $[\mathrm{K}]$ \\
\hline$t$ & dimensional time $[\mathrm{s}]$ \\
\hline$U$ & $\begin{array}{l}\text { dimensional velocity in the } x \text {-direction } \\
{\left[\mathrm{m} \mathrm{s}^{-1}\right]}\end{array}$ \\
\hline$u$ & $\begin{array}{l}\text { nondimensional velocity in the } \\
x \text {-direction, } U L / \alpha\end{array}$ \\
\hline$V$ & $\begin{array}{l}\text { dimensional velocity in the } y \text {-direction } \\
{\left[\mathrm{m} \mathrm{s}^{-1}\right]}\end{array}$ \\
\hline$v$ & $\begin{array}{l}\text { nondimensional velocity in the } \\
y \text {-direction, } V L / \alpha\end{array}$ \\
\hline$X$ & $\begin{array}{l}\text { dimensional coordinate in the } \\
\text { horizontal direction [m] }\end{array}$ \\
\hline
\end{tabular}

$x$ nondimensional coordinate in the

horizontal direction, $X / L$

$Y$ dimensional coordinate in the vertical direction [m]

$y$ nondimensional coordinate in the vertical directon, $Y / L$.

\begin{tabular}{|c|c|}
\hline \multicolumn{2}{|c|}{ Greek symbols } \\
\hline$\alpha$ & thermal diffusivity $\left[\mathrm{m}^{2} \mathrm{~s}^{-1}\right]$ \\
\hline$\beta_{\mathrm{T}}$ & $\begin{array}{l}\text { volumetric coefficient of thermal } \\
\text { expansion }\left[\mathrm{K}^{-1}\right]\end{array}$ \\
\hline$\beta_{\mathrm{S}}$ & $\begin{array}{l}\text { volumetric coefficient of expansion } \\
\text { with solutal }\left[\mathrm{wt}^{0} \%^{-1}\right]\end{array}$ \\
\hline$\phi_{0}$ & initial density gradient, $\beta_{\mathrm{S}}\left|\mathrm{d} C / 2 Y^{\prime}\right|_{0}$ \\
\hline$\eta$ & $\begin{array}{l}\text { characteristic length of layer thickness, } \\
\alpha \Delta T / \phi_{0}\end{array}$ \\
\hline$\Delta C$ & initial solutal difference, $C_{\mathrm{h}}-C_{\mathrm{l}}[\mathrm{wt} \%]$ \\
\hline$\Delta c$ & nondimensional solutal difference \\
\hline$\Delta T$ & initial thermal difference, $T_{\mathrm{h}}-T_{1}[\mathrm{~K}]$ \\
\hline$\Delta \theta$ & $\begin{array}{l}\text { nondimensional temperature } \\
\text { difference }\end{array}$ \\
\hline$\Delta \theta_{\mathrm{b}}$ & $\begin{array}{l}\text { nondimensional temperature } \\
\text { difference between boundaries, } \\
\theta_{\mathrm{h}}-\theta_{1}=1\end{array}$ \\
\hline$\Delta \psi$ & difference of stream function \\
\hline$\phi$ & physical variable of interest \\
\hline$\gamma$ & inclined angle [deg] \\
\hline$v$ & kinematic viscosity $\left[\mathrm{m}^{2} \mathrm{~s}^{-1}\right]$ \\
\hline$\theta$ & $\begin{array}{l}\text { nondimensional temperature, } \\
\left(T-T_{1}\right) /\left(T_{\mathrm{h}}-T_{1}\right)\end{array}$ \\
\hline$\tau$ & nondimensional time, $t \alpha / L^{2}$ \\
\hline$\psi$ & stream function. \\
\hline
\end{tabular}

\section{Subscripts}

$\begin{array}{ll}\text { h } & \text { highest value } \\ 1 & \text { lowest value } \\ 0 & \text { initial value } \\ \max & \text { maximum value } \\ \min & \text { minimum value. }\end{array}$
Superscripts
coordinate after rotation
$\kappa \quad$ iteration index.

imental observations of Lewis et al. [15]. It was analyzed mostly in one-dimensional domain as seen in refs. [1-3], only a few employed the two-dimensional simulation [13, 14].

As to the effect of inclination, the positive angles denote heating from below, while the negative angles denote heating from above. Some authors investigated the double-diffusive problem about a slant, heating plate immersed in a tank with positive angles $[13,16]$, they found the value $R a_{\eta \mathrm{cr}} \times \cos \gamma$ does not change until it is larger than $\gamma=60^{\circ}$, but then it increases rapidly as $\gamma$ increases for positive inclined angles. Paliwal and Chen studied the inclined slot from $\gamma=75^{\circ}$ to $-75^{\circ}$ by the experimental observation [17] and stability analysis [18]. They noted the onset of instability is nonsymmetric with respect to $\gamma=0^{\circ}$, where the secondary flow pattern becomes unstable in a rather 'dramatic' manner at $\gamma=75^{\circ}$. Later, Thangam et al. [19] also demonstrated the nonsymmetric phenomenon in an inclined slot by theoretical analysis. When the cavity is inclined, the flow is less stable near the upper wall than that near the lower 
wall. Even though without heating the buoyancy layers transport fluid up along the downward sidewall and down along the upward sidewall [20]. The heating effect may augment or resist the motion depending on the heating from the lower or upper sidewall. The combined effect causes the solutal gradient near the heating wall to reinforce or reduce and further affect the onset of instability. Besides, as the horizontal layers are formed, the flow is more stable at negative angles, whereas it is more vigorous at positive angles.

All of the above-mentioned papers about the effect of inclined angles are restricted in discussing the onset of instability and the initial solutal gradients are assumed as the sanne values. Research about the transient evolution in the cavity with finite aspect ratio is still lacking. Practically, for an inclined cavity with finite aspect ratio, the length between the highest and lowest points is different at each inclined angle. The present study numerically investigates the doublediffusive convection problem in a finite aspect ratio (equal to 2) rectangular cavity, with inclination angle varied from $\gamma=-75^{\circ}$ to $75^{\circ}$. We focus our study on the $\mathrm{NaCl}-\mathrm{H}_{2} \mathrm{O}$ solution associated with constant temperature and initial solutal difference. In addition to the onset of instability, the flow types and mass transfer characteristics during a long period of time are systematically discussed. Also, we will compare the numerical results with those of the flow visualization experiments by shadowgraph method.

\section{NUMERICAL SIMULATION}

\section{Mathematic formulation}

The fluid is assumed to remain laminar, two-dimensional and incompressible with constant fluid properties, except for the density change with temperature and salinity that induces the buoyancy forces, i.e. the adoption of the Floussinesq approximation. Hence, the governing equations can be written in the following dimensionless forms :

continuity equation

$$
\frac{\partial u}{\partial x}+\frac{\partial v}{\partial y}=0
$$

momentum equations

$$
\begin{array}{r}
\frac{\partial u}{\partial \tau}+u \frac{\partial u}{\partial x}+v \frac{\partial u}{\partial y}=-\frac{\partial p}{\partial x}+\sin \gamma \\
\operatorname{RaPr}\left(\theta-R_{\rho} c\right) \\
+\operatorname{Pr}\left(\frac{\partial^{2} u}{\partial x^{2}}+\frac{\partial^{2} u}{\partial y^{2}}\right) \\
\frac{\partial v}{\partial \tau}+u \frac{\partial v}{\partial x}+v \frac{\partial v}{\partial y}=-\frac{\partial p}{\partial y}+\cos \gamma \operatorname{RaPr}\left(\theta-R_{\rho} c\right) \\
+ \\
\operatorname{Pr}\left(\frac{\partial^{2} v}{\partial x^{2}}+\frac{\partial^{2} v}{\partial y^{2}}\right)
\end{array}
$$

energy equation

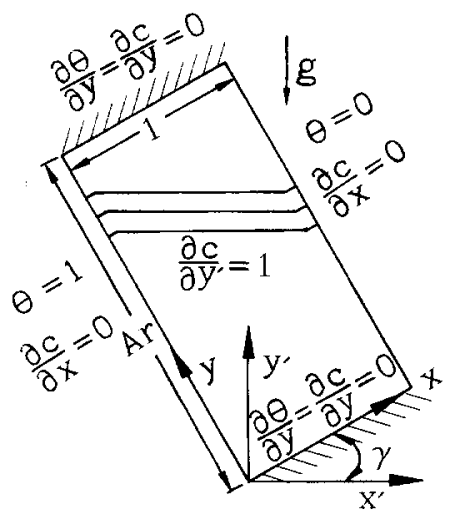

Fig. 1. Schematic of the physical domain.

$$
\frac{\partial \theta}{\partial \tau}+u \frac{\partial \theta}{\partial x}+v \frac{\partial \theta}{\partial y}=\frac{\partial^{2} \theta}{\partial x^{2}}+\frac{\partial^{2} \theta}{\partial y^{2}} ;
$$

concentration equation

$$
\frac{\partial c}{\partial \tau}+u \frac{\partial c}{\partial x}+v \frac{\partial c}{\partial y}=\frac{1}{L e}\left(\frac{\partial^{2} c}{\partial x^{2}}+\frac{\partial^{2} c}{\partial y^{2}}\right)
$$

The nondimensional quantities introduced are defined as

$$
\begin{gathered}
u=\frac{U L}{\alpha} \quad v=\frac{V L}{\alpha} \quad x=\frac{X}{L} \\
y=\frac{Y}{L} \quad \tau=\frac{t \alpha}{L^{2}} \quad p=\frac{P L^{2}}{\rho \alpha^{2}} \\
\theta=\frac{T-T_{1}}{T_{\mathrm{h}}-T_{\mathrm{l}}} \quad c=\frac{C-C_{\mathrm{l}}}{C_{\mathrm{h}}-C_{\mathrm{l}}} \quad A r=\frac{H}{L} \\
\operatorname{Pr}=\frac{\nu}{\alpha} \quad R a=\frac{g \beta_{\mathrm{T}} \Delta T L^{3}}{\alpha \nu} \\
R a_{\mathrm{s}}=\frac{g \beta_{\mathrm{s}} \Delta C L^{3}}{\alpha \nu} \quad R_{\rho}=\frac{R a}{R a_{\mathrm{s}}} \quad L e=\frac{\alpha}{D} .
\end{gathered}
$$

Here, $A r$ is the aspect ratio, $P r$ is the Prandtl number, $L e$ is the Lewis number, $R a$ and $R a_{\mathrm{s}}$ are the thermal and solutal Rayleigh numbers, and $R_{\rho}$ represents the relative magnitude of solutal and thermal buoyancy forces.

The schematic diagram of the physical domain is shown in Fig. 1. Initially, the cavity is filled with fluid of a linear solutal gradient in the gravitational direction. Suddenly, one sidewall has a higher temperature imposed $T_{\mathrm{h}}$ and the opposite sidewall remains at the initial temperature $T_{1}$, while the top and bottom walls are kept insulated. All the initial and boundary conditions are as follows:

$$
\begin{gathered}
u=v=\theta=0, \quad c=1-\frac{y^{\prime}}{\sqrt{1+A r^{2}}} \text { at } \tau=0 \\
u=v=\frac{\partial c}{\partial n}=0 \quad \text { on all boundaries } \\
\theta=1 \quad \text { on } x=0, \quad \theta=0 \text { on } x=1
\end{gathered}
$$




$$
\frac{\partial \theta}{\partial y}=0 \text { on } y=0 \text { and } A r .
$$

Because the coordinate of computational domain $(x-y)$ may be different from the fixed coordinate $\left(x^{\prime}-y^{\prime}\right)$, the initial solutal conditions in equation (6) needs to be transferred to the $(x-y)$ domain. The following equation can be used for the positive angles :

$$
c(x, y)=1-\frac{\sqrt{x^{\prime 2}+y^{\prime 2}} \cos \left(\cos ^{-1}\left(\frac{y^{\prime}}{\sqrt{x^{\prime 2}+y^{\prime 2}}}\right)-\gamma\right)}{\sqrt{1+A r^{2}} \cos \left(\cos ^{-1}\left(-\frac{A r}{\sqrt{1+A r^{2}}}\right)-\gamma\right)}
$$

and, for negative angles, equation (10) can also be employed after changing the heating surface and mirroring the results with respect to the axis of $x=\cos \gamma$.

Besides, there is another parameter adopted for discussing the onset of instability, that is, the modified Rayleigh number

$$
R a_{\eta}=\frac{g \beta_{\mathrm{T}} \Delta T \eta^{3}}{\alpha \nu}\left(=R a\left(\frac{A r}{R_{p}}\right)^{3}\right)
$$

where

$$
\eta \equiv \frac{\beta_{\mathrm{T}} \Delta T}{\phi_{0}}\left(=\frac{H}{R_{p}}\right)
$$

The length scale $\eta$ denotes the elevation that the fluid parcel can rise after their density is changed [5, 10] and $\phi_{0}=\beta_{\mathrm{s}}\left|\mathrm{d} C / \mathrm{d} Y^{\prime}\right|_{0}$.

The calculated heat transfer at the wall is presented by the local or average Nusselt number, $N u$ or $\overline{N u}$, which are defined respectively, as

$$
N u=-\frac{A r}{\Delta \theta_{\mathrm{b}}}\left(\frac{\partial \theta}{\partial x}\right)_{x=0} \quad \overline{N u}=\frac{1}{A r} \int_{y=0}^{A r} N u \mathrm{~d} y .
$$

\section{Numerical method and grid test}

Equations (1)-(5) are discretized using the weighing function scheme [22] and are solved using the finite difference method, i.e. the APPLE algorithm developed by Lee and Tzong [23].

The distribution of grid points gradually concentrates toward the boundaries for analyzing the boundary layer. The grid net affects the solution of simulation, especially in the $y$-direction. Hence, there should be enough grids to analyze the multi-layered structure [11]. We use 60 grid points in the $x$-direction, somewhat larger than the grids of Lee and Hyun [10]. Different grid meshes of $65,75,85,95$ and 105 in the $y$-direction are employed to make the grid test, and the results are presented in Table 1. As seen from Table 1, when the grid meshes in the $y$-direction were chosen as 75 , the cell numbers are 9 at an early stage, which are less than that with denser grid meshes. The
Table 1. Grid test at different times

\begin{tabular}{rccccc}
\hline & $\tau=0.2$ & $\tau=0.4$ & $\tau=0.6$ & $\tau=0.8$ & $\tau=1.0$ \\
\hline 105 & 10 & 9 & 5 & 5 & 5 \\
95 & 10 & 9 & 5 & 5 & 5 \\
85 & 10 & 9 & 5 & 5 & 5 \\
75 & 9 & 7 & 5 & 5 & 4 \\
65 & 8 & 6 & 4 & 4 & 4 \\
\hline
\end{tabular}

cell evolution is also faster than the other cases with larger grid meshes. Therefore, in our calculations, we use grid meshes of 95 in the $y$-direction, considering both the accuracy and economy of computing. We have adopted the following convergence criteria at each time step:

$$
\frac{\left|\phi^{\kappa}-\phi^{\kappa+1}\right|}{\left|\phi_{\max }^{\kappa+1}-\phi_{\min }^{\kappa+1}\right|} \leqslant 10^{-5}
$$

where $\phi$ indicates the physical variable of interest and superscript $\kappa$ denotes the iteration index.

\section{RESULTS AND DISCUSSION}

In this study, we use the parameters of $R a=1 \times 10^{7}$, $R_{\rho}=5$ and $A r=2$ within the range $-75^{\circ} \leqslant \gamma \leqslant 75^{\circ}$. The modified Rayleigh number $R a_{\eta}$ calculated from $R a, R a_{\mathrm{s}}$ and $A r$, is $6.4 \times 10^{5}$ for $\gamma=0^{\circ}$, which exceeds the critical value $15000 \pm 2500$ by a great amount. The mechanism of the onset of instability and the layered structure in our study can also be divided into the horizontal layer structure $\left(\gamma<75^{\circ}\right)$ and the wellmixed fluid layer structure $\left(\gamma \geqslant 75^{\circ}\right)$. For convenience, we shall discuss them separately.

\section{Onset of instability}

We look into some features which occur close to the onset of instability, as shown in Fig. 2, with stream function, $\psi$, defined as $u=\partial \psi / \partial y$ and $v=-\partial \psi / \partial x$. At very early time, the cells are generated near the bottom and top walls, and then, some simultaneous cells appear in the middle portion and intrude toward the interior region. The former is due to the end effects of the bottom and top walls [21], and the latter is the secondary flow due to the onset of instability $[5,10]$. Although the rotation of the major cells is clockwise, the data of negative stream function in the interfaces and the near-stagnation regions reveal that some secondary cells rotate counterclockwise. The secondary cells are so weak that they cannot be clearly captured in the plots. This is consistent with the theoretical predictions reported in refs. $[4,19]$.

The onset of instability is more pronounced at the negative angles than the positive ones as seen from Fig. 2. This nonsymmetric phenomenon is attributed to the solutal gradient adjacent to the boundaries, which are different from that in the interior region owing to the nondiffuse boundary conditions. Accordingly, the isosolutal lines adjacent to the upper side- 


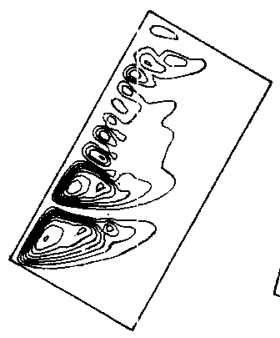

(1)

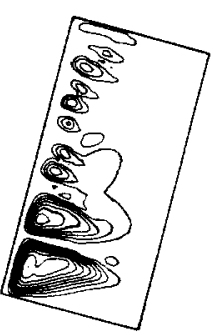

(2)

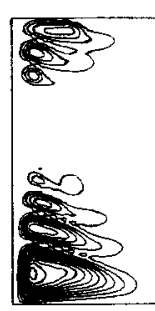

(3)

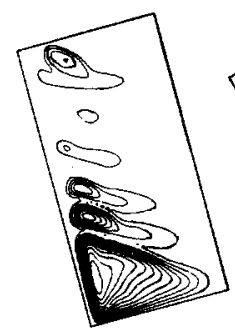

(4)

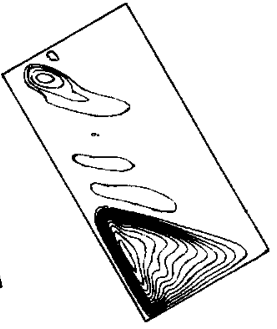

(5)

Fig. 2. Stream line plots at about the onset of instability $(\tau=0.01)$, where $\Delta \psi=-2$ for all cases.

wall curve upward, while the isosolutal lines adjacent to the lower sidewall curve downward. This phenomenon causes the density variation and therefore, the buoyancy layers induce the flow up and down along the sidewalls. If the heating is imposed from below, the thermal boundary layer enhances the flow motion and the solutal gradient in the vicinity of the lower sidewall such that the onset of instability is slower. However, if the heating is from above, the thermal boundary layer reduces the flow motion and the solutal gradient in the vicinity of the upper sidewall such that the onset of instability is sooner, as reported in refs. [17-19]. It is demonstrated in this study by solving the full $N-S$ equations in the cavity with finite aspect ratio.

The onset of instability would be the combined effect of solutal and thermal gradients. All of our discussions will be focused on the same initial solutal difference with linear distribution, but the initial solutal gradient is different for various inclined angles.

\section{Stream lines distritution}

We shall scrutinize the gross features of the flow at positive angles (including $\gamma=0^{\circ}$ ) first and the negative angles later.

When the onset of instability has occurred for a period of time, the cell structure develops in the cavity, as seen in Fig. 3(a). The fluid parcel rising from the sidewall continuously loses heat to the cold ambient, and so, the density increases. Hence, the cell intrusion is slightly lower than the horizontal direction for all the inclined angles. When the angle increases, the heating-from-below effect is more and more notable that the fluid parcel near the left bottom corner thrusts more and more highly along the sidewall. This makes the bottom cell more and more vigorous and reduces the cell numbers accordingly. On the other hand, because the end effect of the top wall is reducing, the top cell becomes weaker and weaker as the angle increases [see Fig. 3(a)].

In the process of the cells' evolution, merging occurs between the cells, and the cell numbers decrease in every angle. At $\tau==0.1$ [Fig. 3(b)], the layered structure is well-developed, i.e. all the simultaneous layers show a similar structure independent of the inclined angles. The horizontal interfaces separate the layers and the shear force comes from the circulations in the two layers acting on them. Because heat is also subtracted from the right wall, convection is reinforced so that the merging is also accelerated from top and bottom. The bottom layer is more unstable and occupies the larger portion of the cavity as the angle increases. It is seen from Fig. 3(a,b) that the inclined angle greatly influences the flow type, especially for the bottom layer. Hence, at large angles, the simultaneous layers could become the minor feature.

The stream function plots at negative angles are presented in Fig. 4. Although the onset of instability at the negative angles is sooner than that at the positive ones for the same $|\gamma|$, the thermally driven flow is weaker at the negative angles, as shown in Fig. 4(a). The simultaneous cells are larger, but are not so complete as that at the positive angles. As compared with the positive angles, some interesting features are observed during the cell evolution. Firstly, the bottom cell is not able to occupy the bottom corner, but behaves as if there is a horizontal boundary above the bottom corner because the density of the lowest point adjacent to the heating wall is smaller than that lower than it such that the motion of fluid parcel cannot reach the bottom corner. Secondly, at the upper part of the heating surface, the cells cannot develop further owing to the opposite insulated wall and therefore, are restricted to the upper left corner. For a long period of time they remain unchanged. The most striking case is at $\gamma=-75^{\circ}$, as presented in Fig. 4(b), due to the weak heating effect and insulted top wall, the fluid parcel comes from the heating wall and cannot arrive at the opposite wall so that the cells show a sharper front. Hence, the maximum value of the stream function decreases as the angle decreases [see Fig. 4(a)]. However, the merging process will retard the convection, as shown in Fig. 4(b).

Some typical cases at both positive and negative angles are depicted in Fig. 5 when the flow approaches a steady state. For $\gamma \geqslant 0^{\circ}$, the flow types are similar to the pure natural convection with homogeneous solutal distribution; while for $\gamma<0^{\circ}$, the solutal distribution is not homogeneous in the cavity. As discussed above, at negative angles, the part of convection cannot occupy the whole region. Therefore, the solutal distri- 
(a)

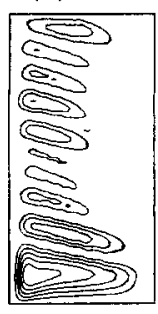

(1)

(b)

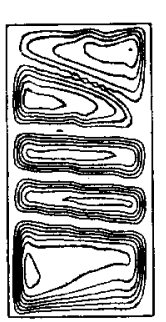

(1)

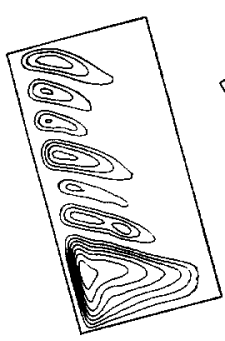

(2)

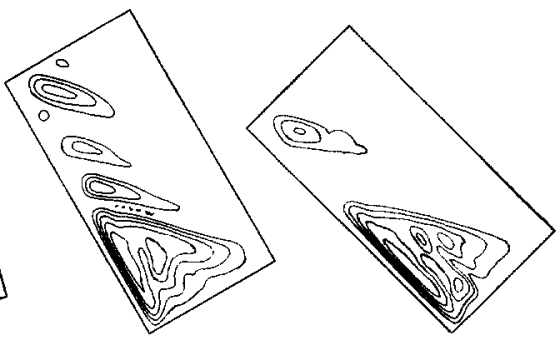

(3)
(4)

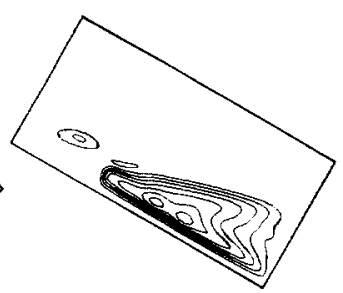

(5)

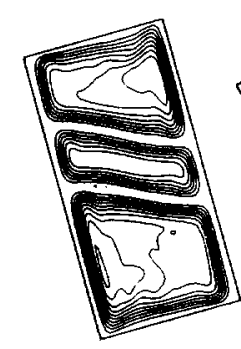

(2)

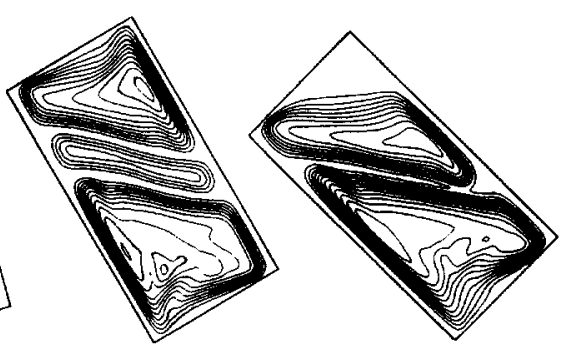

(3)

(4)

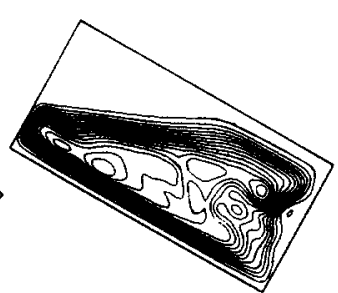

(5)

Fig. 3. (a) Stream line plots for positive angles at $\tau=0.02$, where $\Delta \psi=-4$ for all cases. (b) Stream line plots for positive angles at $\tau=0.10$, where $\Delta \psi=-4$ for all cases.

bution shows a different type in the weak convection region at the top corner and the no convection region at the bottom corner. As the angle increases, the time steadily increases at first then decreases. This is because of the merging with different numbers of layers. It is interesting to note that the tendency of the time to the steady state are roughly opposed to that of the onset of instability. That is, at the negative

(a)

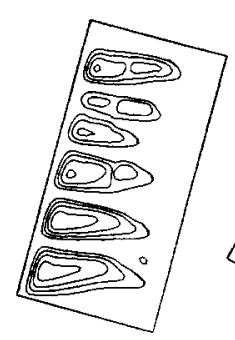

(1)

(b)

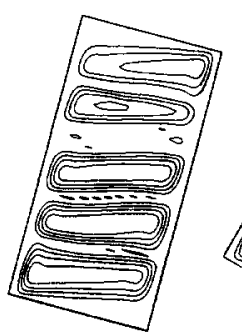

(1)

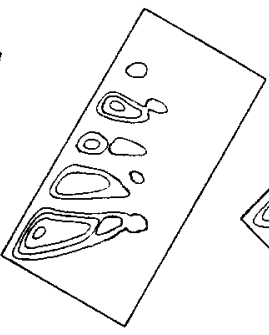

(2)

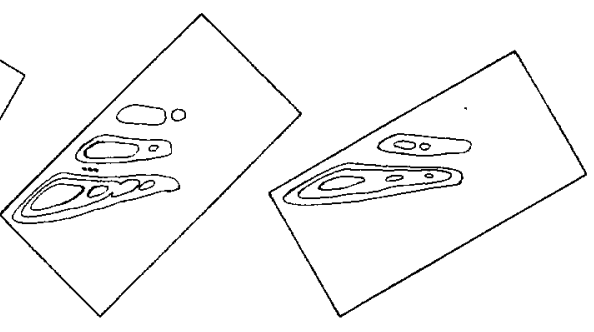

(4)

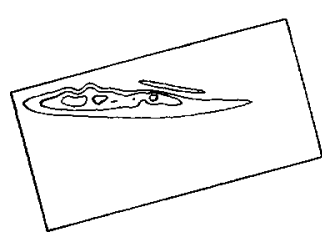

(5)

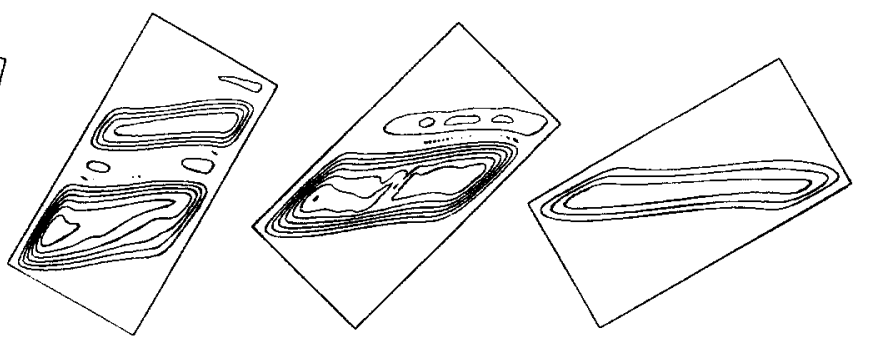

(3)
(4)

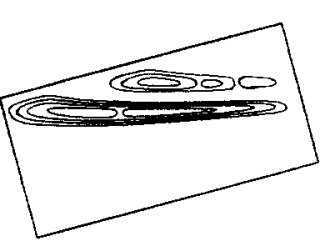

(5)

Fig. 4. (a) Stream line plots for negative angles at $\tau=0.02$, where $\Delta \psi=-1$ for $\gamma=-75^{\circ}$ and $\Delta \psi=-4$ for other cases. (b) Stream line plots for negative angles at $\tau=0.10$, where $\Delta \psi=-1$ for $\gamma=-75^{\circ}$ and $\Delta \psi=-4$ for other cases. 


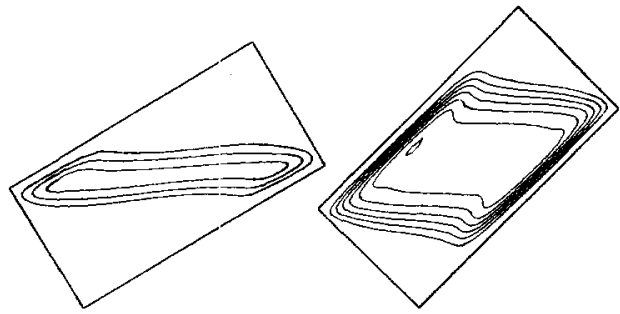

(1)

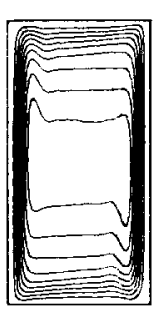

(3)

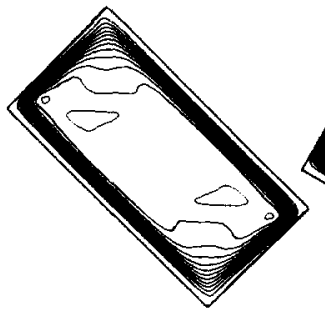

(4)

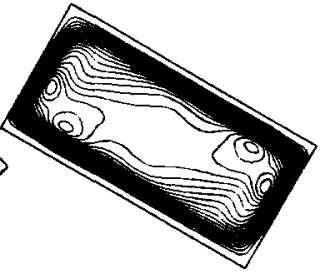

(5)

Fig. 5. Stream line plots for some inclined angles after a long time. The times from left to right are $\tau=0.2$, $0.4,0.7,0.6,0.4$, where $\Delta \psi=-6$ for all cases.

angles the steady state is reached sooner than the positive ones for the same $|\gamma|$.

\section{Isotherms distribution}

Figures $6(a, b)$ and $7(a, b)$ are the isotherm plots for different angles. At $\tau=0.02$ [Fig. 6(a)], the cell intrusion is progressing. Because only one boundary is heating, the cells are associated with the vivid convection, while the other portion remains static. Due to the recirculation within each cell a wavy temperature distribution exists. The most vigorous convection is exhibited in the bottom cell such that the temperature distribution is almost uniform there and the thermal boundary layer is evident. A stronger convection also occurs in the top cell. As the angle is increased, the convection is more and more reinforced at the bottom, but is more and more retarded at the top. The simultaneous cells evolve with a similar tendency of temperature distribution. At the interfaces of the cells the isotherms are denser. There the heat transfer is dominated by conduction. The heat flux is largest at the heating sidewall, and induces the entire convection motion. It is interesting to point out that the rate of heat transfer adjacent to the top wall is larger than that adjacent to the bottom wall, even though the bottom cell is more vigorous in strength for all cases [see Fig. 6(a) the first isothermal line from the right wall]. At $\tau=0.1$ [Fig. 6(b)] the layered structure is well developed for all angles and the conduction region has disappeared. The heat transferred from the left wall and subtracted from the right wall enhances the convection motion. When the heat transfer is large enough, it will destroy the stable solutal interfaces to initiate the merging process, the temperature distribution will be somewhat random, but returns to wavy distribution as the merging ends. When the angle is increased, the temperature field becomes more and more unstable [see Fig. 6(b)].

(a)

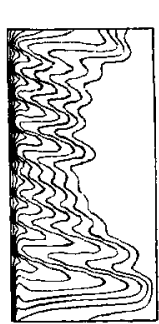

(1)

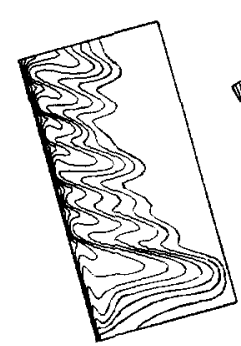

(2)

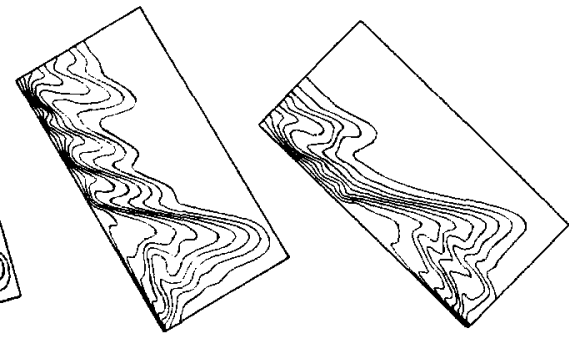

(3)
(4)

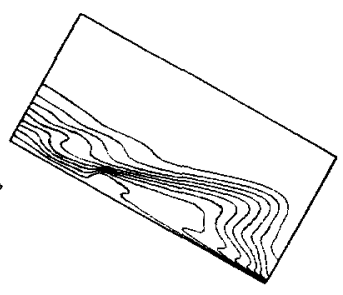

(5)

(b)

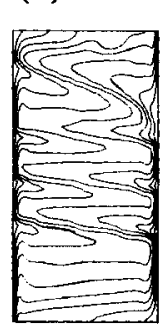

(1)

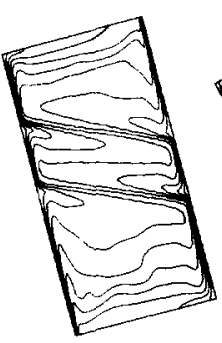

(2)

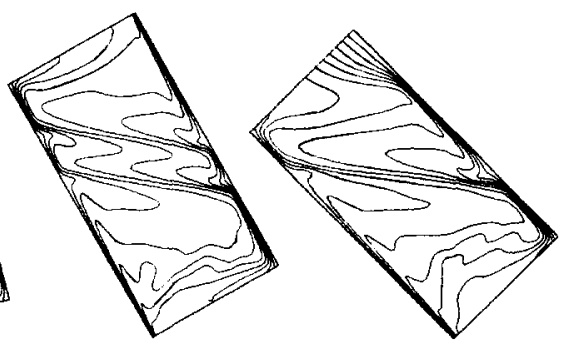

(3)
(4)

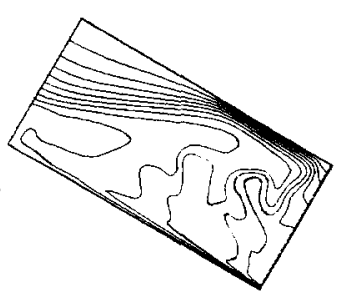

(5)

Fig. 6. (a) lsotherm plots for positive angles at $\tau=0.02$, where $\Delta \theta=0.1$ for all cases. (b) Isotherm plots for positive angles at $\tau=0.10$, where $\Delta \theta=0.1$ for all cases. 
(a)

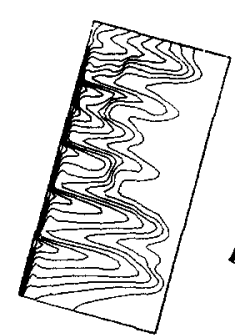

(1)

(b)

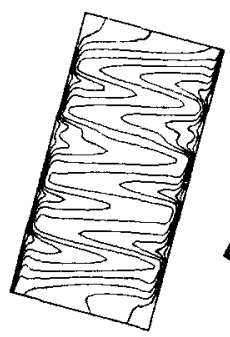

(1)

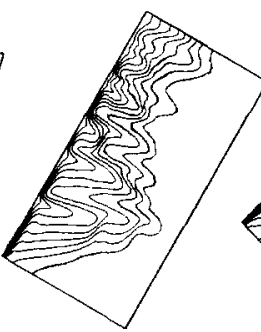

(2)

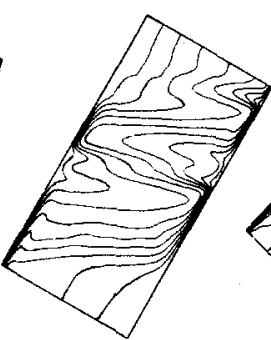

(2)

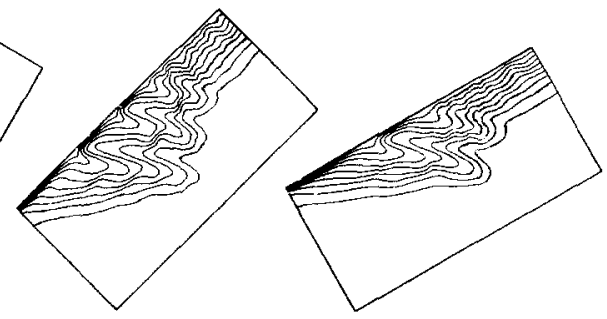

(3)

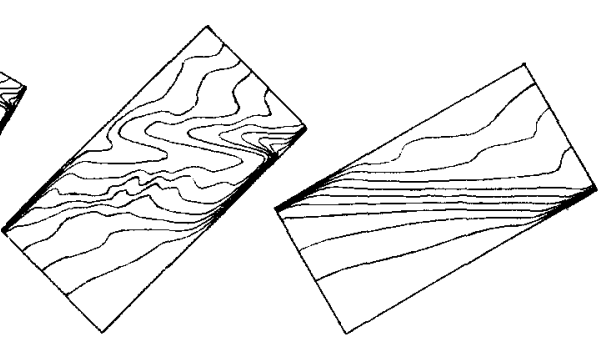

(3)
(4)

(4)

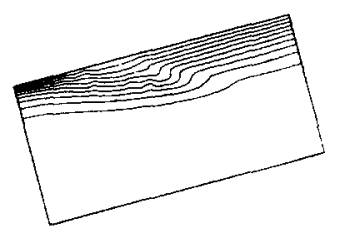

(5)

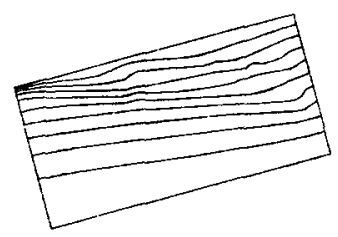

(5)

Fig. 7. (a) Isotherm plots for negative angles at $\tau=0.02$, where $\Delta \theta=0.1$ for all cases. (b) Isotherm plots for negative angles at $\tau=0.10$, where $\Delta \theta=0.1$ for all cases.

At negative angles, as shown in Fig. $7(a, b)$, the tendencies of temperature distribution in the cells and layers are similar to that at the positive angles, but are weaker in magnitude. Since the cells cannot fill the whole region, the conduction dominates accordingly at the top and bottom corners, and increases as the angle decreases. Similar to that at the positive angles, the rate of heat transfer adjacent to the top wall is larger than that adjacent to the bottom wall.

\section{Isosolutal lines distribution}

The isosolutal line distributions are demonstrated in Figs. 8 and 9. The solute becomes more uniform in the cell region and remains the linear distribution outside of the cell regions [see Fig. 8(a)]. Since the thermal diffusivity is larger than the mass diffusivity, the solutal distribution responses slowly to the cell propagation. At $\tau=0.1$ [Fig. 8(b)], the layered structure is well developed and it is exhibited that there is the sharp solutal difference in the interfaces. Not only is the heat transferred through the interfaces, but also the mass is carried up to the upper layers and the interfaces become thinner and thinner. It is interesting that some isosolutal lines at the interface are zigzagged. This phenomenon is concerned with the instability of merging. When the interface becomes very thin, the shear forces adjacent to the interface might lead to the onset of Kelvin-Helmholtz instability. Since the merging begins near the walls, due to the thermal boundary layers established along the side- walls, the interface is not able to keep the horizontal structure anymore and becomes inclined. The interface breaks up near the walls, and then is rapidly transferred to the inner region. The entire process is similar to the two-layer system, as delineated in ref. [25]. Due to the heating-from-below effect, the merging process is faster as the angle increases.

For the negative angles, at $\tau=0.02$ [Fig. 9(a)], the mass transfer in the cell region is weaker than that at the positive angles. As the layers are well developed [Fig. 9(b)], the solutal gradient at right bottom corner is still linear and does not vary for a long period of time. However, it is not entirely linear at the left upper corner due to the existence of cells with very weak convection, as discussed previously.

\section{Heat transfer characteristics}

Some typical cases of local Nusselt number at the heated wall at $\tau=0.08$ are shown in Fig. 10. The distribution of heat transfer at the sidewall is wavy. The distribution is relevant to the layered structure, accordingly. The periodic results at $\gamma=0^{\circ}$ is consistent with the experimental finding of ref. [6] and the numerical results of refs. $[10,11]$. Owing to the inclined effect, the number of waves at other angles are fewer than that at $\gamma=0^{\circ}$ and the wavy distribution is concentrated at the above region for positive angles. However, the layered structure is mainly evolutionary at a lower region for negative angles.

The time variations of the average Nusselt number 
(a)

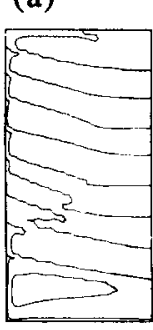

(1)

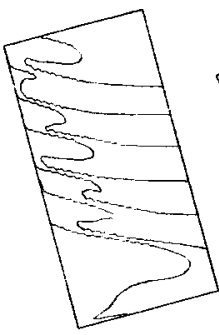

(2)

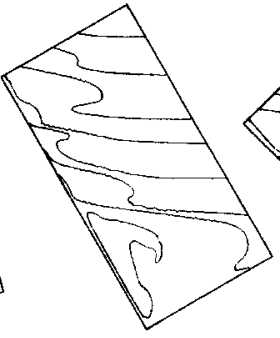

(3)

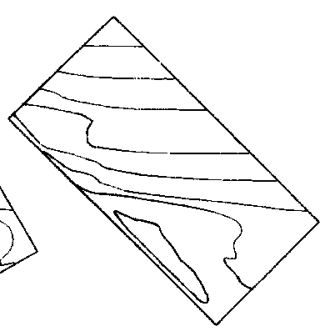

(4)

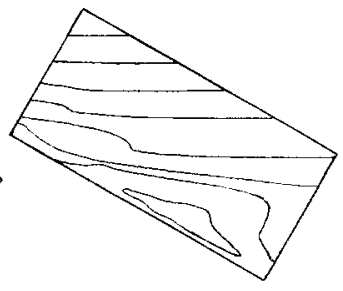

(5)

(b)

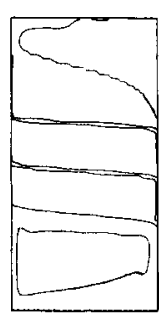

(1)

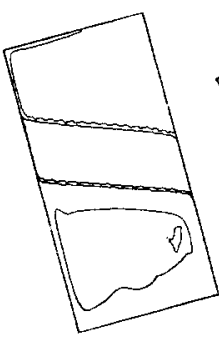

(2)

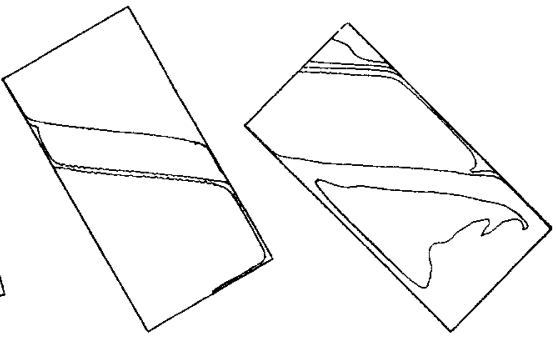

(3)
(4)

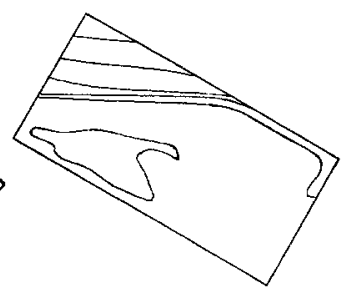

(5)

Fig. 8. (a) Isosolutal plots for positive angles at $\tau=0.02$, where $\Delta c=0.1$ for all cases. (b) Isosolutal plots for positive angles at $\tau=0.10$, where $\Delta c=0.1$ for all cases.

at some inclined angles are shown in Fig. 11. There is a rugged trend in plots that is different from the smooth distribution of pure natural convection or conduction. Moreover, the distribution is between these two modes [12]. It reveals that the layered structure greatly influences the heat transfer characteristics. The average Nusselt number tends to descend at early time as the cells are generated and propagating from the

(a)

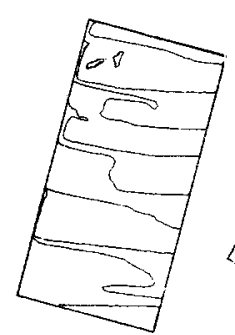

(1)

(b)

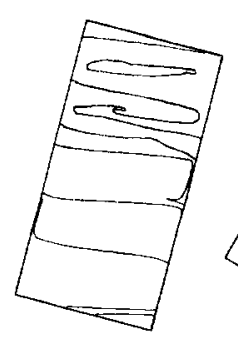

(1)

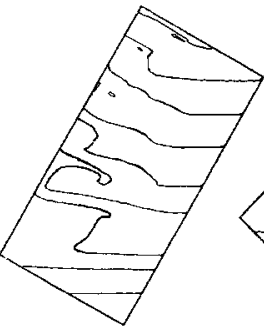

(2)

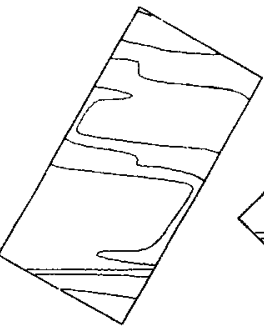

(2)

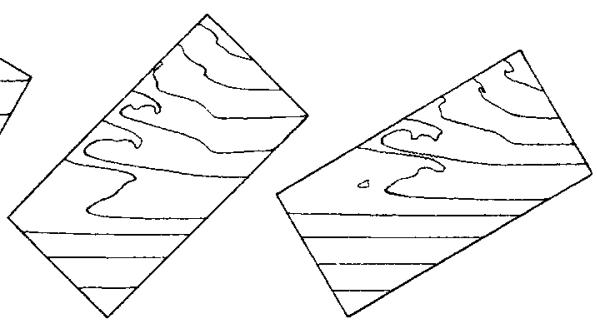

(4)

(3)

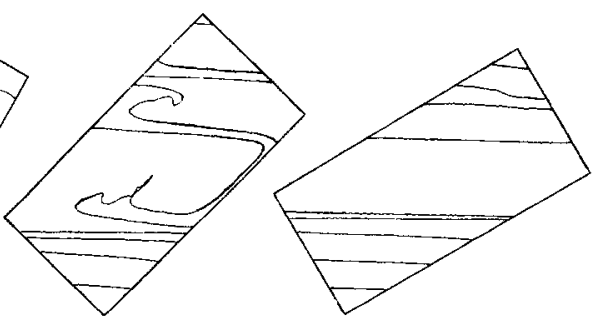

(3)

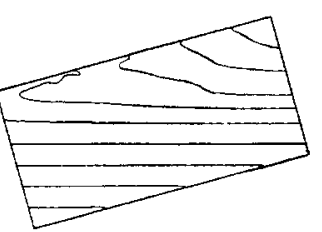

(5)

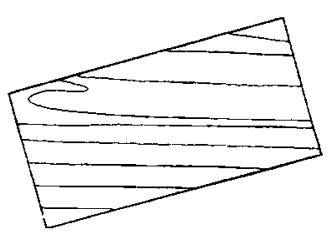

(5)

Fig. 9. (a) Isosolutal plots for negative angles at $\tau=0.02$, where $\Delta c=0.1$ for all cases. (b) Isosolutal plots for negative angles at $\tau=0.10$, where $\Delta c=0.1$ for all cases. 


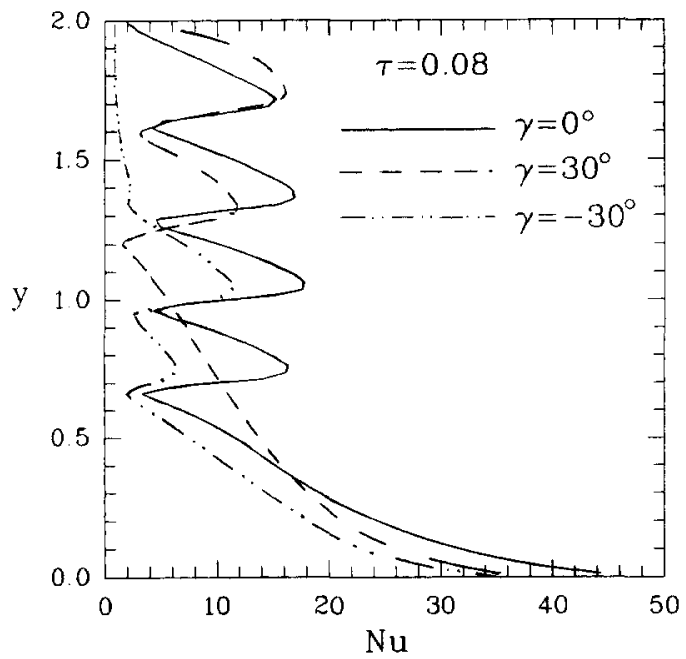

Fig. 10. Plot of the local Nusselt number at some inclined angles at $\tau=0.08$.

heated wall and later it rises abruptly as the cells transform into the layered structure. It is noted that the merging between the simultaneous cells does not lead to a marked change of the average Nusselt number. The plots have significant variations as layers merge. Even without merging, the flow field is not entirely stable in the layers and it will vibrate with respect to time. There is a drop of it and the instability becomes more striking as the Kelvin-Helmholtz instability is incorporated. It reaches to a minimum when the interface breaks up. This process will repeat again and again until steady state is achieved as presented in Fig. 11. The whole behavior is similar to the experimental results in ref. [24]. Due to a constant heat flux condition, the steady state does not exist in their results.

Due to the heating-from-below effect, the variations at positive angles are more vigorous than that at negative ones for the same $|\gamma|$, as discussed previously. The corresponding cell numbers are indicated below the distribution. The cell number decreases as the angle increases and steady state is achieved more rapidly at positive angles. At negative angles, the cell numbers increase as the angle increases and it takes a longer time to get to a steady state at a larger angle. We observe also that at $\gamma=-60^{\circ}$ the layered structure is so weak that the curve is nearly smooth until to the steady state. At the steady state the average Nusselt numbers at the positive angles are the same as that of the natural convection. However, they are significantly smaller at negative angles because the flow cannot move over the entire cavity.

\section{Results of $\gamma=75^{\circ}$}

The most interesting case is at $\gamma=75^{\circ}$. At this angle, the other kind of instability could be onset. It is mainly due to the parallel and opposed thermal and solutal gradients. The typical case is the salt stratified fluid layer with heating from bottom, as depicted in refs.
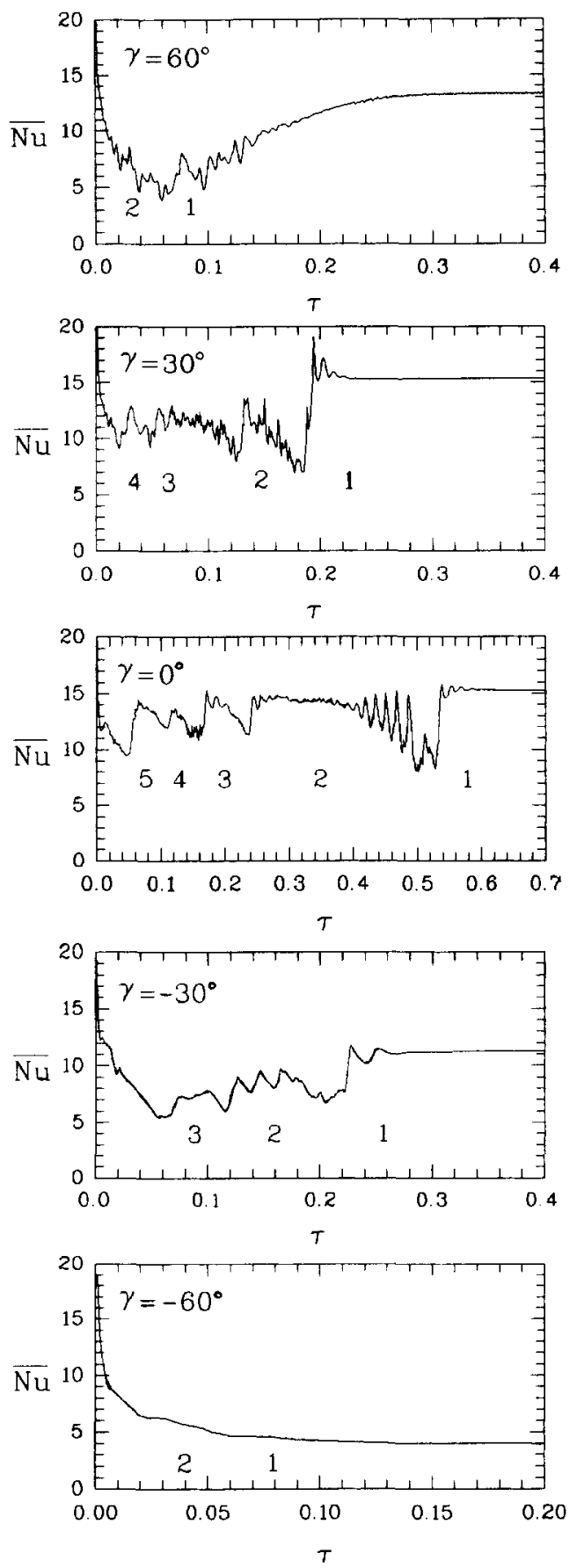

Fig. 11. Transient evolution of average Nusselt number at some inclined angles.

$[2,3,13-15]$. The bottom cell is replaced by some pairs of vortexes rotated with different sense [see Fig. 12(1)]. This instability phenomenon and that discussed previously influence mutually.

The vortexes extend successively along the heating surface [see Fig. 12(2)]. When they come up to the top wall, the vortexes rotating in clockwise direction are reinforced rapidly and severely compress the counterclockwise ones, as shown in Fig. 12(3). At $\tau=0.2$ [Fig. 12(4)], there are only two vortexes rotated with 

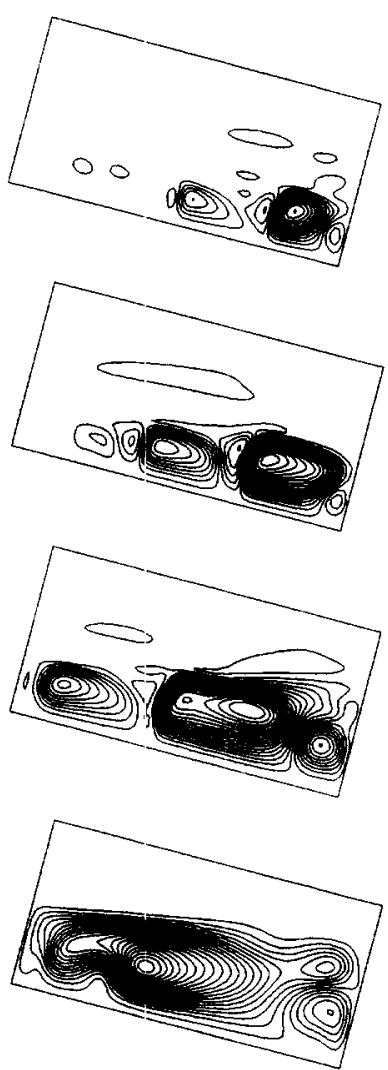

Fig. 12. Transient evolution of stream line plots at $\gamma=75^{\circ}$. Times are $\tau=0.02,0.04,0.10,0.2$ where $\Delta \psi=-4$ for (1), (2), (i) and $\Delta \psi=-6$ for (4).

different sense. Even though the convection is more vigorous than at the other angles, the growth in the $x$-direction is relatively slower. Unlike the flow visualization result in ref. [17], with $A r=25$, we do not find the simultaneous cells in our plots of stream lines. This is due to the small aspect ratio $(A r=2)$ in our study. There are some interesting special phenomena to be found in ref. [17]. There was a blob of fluid thrusting from the bottom to the interface which may come from the repression of the vortexes rotating with different sense [see Fig. 12(2,3)].

The corresponding temperature distribution also behaves differently [see Fig. 13(a)]. Some plumes thrust from the lower portion of the sidewall. Above the plumes and also in the region near the upper part of sidewall, heat conduction is dominant. The unstable effect leads to a more vigorous convection than that at the other angles. At $\tau=0.1$, the entire heating sidewall generates pronounced convective motion and the temperature is uniform there. However, the effect of conduction still dominates in the other part of the cavity. Due to the solutal effect, the interface of the mixed region is approximately horizontal. The solutal distribution shows a vortex occupied region with strong convection and a conduction dominant region in the upper part [see Fig. 13(b)].
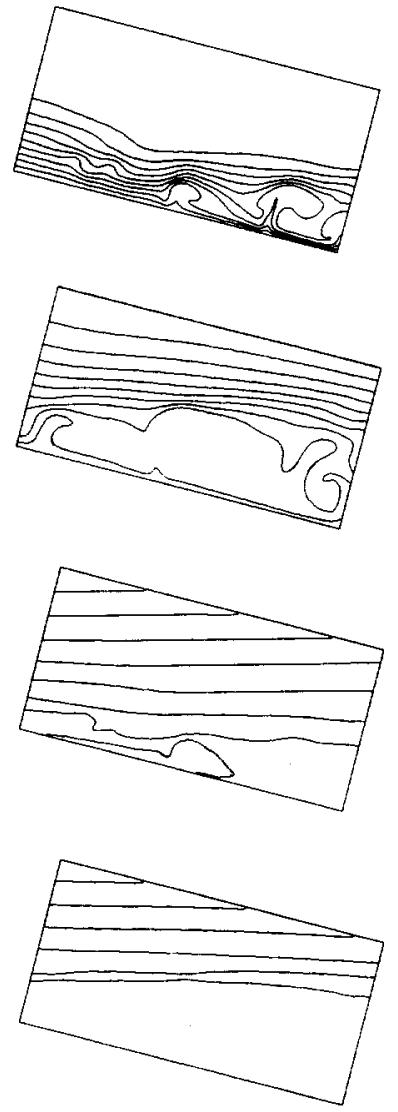

Fig. 13. (a) Isotherm plots at $\gamma=75^{\circ}$ and (b) isosolutal plots at $\gamma=75^{\circ}$. Times from top to bottom for both (a) and (b) are $\tau=0.02$, and $\tau=0.10 ; \Delta c=0.1$ for all cases.

\section{Final layer thickness}

The final layer thickness $h$ is also an important parameter. As studied previously in refs. [5-8, 10], its general definition is the average size of the simultaneous layers after the temperature difference has reached the prescribed value. It was thought that the cells should steadily approach the opposite wall without significant merging phenomena. However, even though the temperature difference has been at the prescribed value for a long time, the merging between the simultaneous cells still exists. This makes the proper estimation difficult and the obtained value is also not reliable. From the plots of flow field at different time and the distribution of average Nusselt number we find that there is a more stable size as the merging between the simultaneous cells has been completed. This time corresponds to the first minimum of the plots in Fig. 11. At this time the average size of all the simultaneous layers (except to the top and bottom cells) was measured.

Accordingly, after careful measurements we obtained the average values at different inclined angles and reported them in Table 2. From Table 2, the value at $\gamma=0^{\circ}$ is equal to $0.675 \eta$, which agrees very well with the experimental results of refs. [5-8]. As to the other angles, the layer developed is found thicker as 
Table 2. The final layer thickness for different inclined angles

\begin{tabular}{cccccc}
\hline & $\gamma=-30^{\circ}$ & $\gamma=-15^{\circ}$ & $\gamma=0^{\circ}$ & $\gamma=15^{\circ}$ & $\gamma=30^{\circ}$ \\
\hline$h$ & $0.64 \eta$ & $0.636 \eta$ & $0.675 \eta$ & $0.682 \eta$ & $0.72 \eta$ \\
\hline
\end{tabular}

the angle is increased. However, as the angle decreases, the thickness becomes slightly thinner at first and then increases again. For the same $|\gamma|$, it is found to be nonsymmetric, as in the experimental results of refs. $[17,18]$. The agreement also demonstrates the accuracy of our calculations. Although there are more cells propagating toward the opposed wall, the merging process is also more vigorous between the simultaneous cells at positive angles. In contrast, at the negative ones, the merging process between the simultaneous cells is less striking. Therefore, the layer thickness at negative angles is smaller than that at the positive angles for the same $|\gamma|$.

\section{EXPERIMENTAL VERIFICATION}

To verify the results of the numerical simulation, we carried out some flow visualization experiments. The technique used is the shadowgraph method, which has been used by many researchers [5-7, 11, $16-19,24]$. The tank used was $8 \mathrm{~cm}$ high $\times 4 \mathrm{~cm}$ wide $\times 3 \mathrm{~cm}$ deep. It consisted of acrylic plates and two copper sidewalls. The counter-flow passages were designed at the back of the sidewalls for keeping the uniform temperature. Four $\mathrm{T}$ type thermalcouples were installed at each copper plate and the temperature was recorded automatically with a recorder. Two constant temperature baths with PID controller were used to maintain the isothermal condition. The tank was installed on an inclined stand where the inclination angle could be adjusted. The working medium was $\mathrm{NaCl}-\mathrm{H}_{2} \mathrm{O}$. A $40 \mathrm{~mW}$ He-Ne laser was used as the light source. The linear solutal distribution was prepared by the double-bucket method. Because the solutal difference is quite small in the case studied and the cell generation seems sensitive to the initial solutal gradient, the filling process must be done under carefully controlled condition. We controlled the filling process by adjusting the flow rate of the solution dropping into the tank. The linear condition obtained by the above method had never been tested by the holography interferometry method and the deviation is within $3 \%$. The solutal difference was obtained by converting the measured data via the densitometer (it is accurate to $\pm 0.001 \mathrm{~g} \mathrm{~cm}^{-3}$ ). The physical properties used were adopted from the International critical table. After the filling process, the experiment is started and the variation of density field could be recorded by the camera.

The flow visualization results by shadowgraph at positive angle $\gamma=30^{\circ}$ and negative angle $\gamma=-30^{\circ}$ are shown as an example in Figs. 14 and 15, respectively. It demonstrates that the numerical predictions of cell progress [Figs. 14(a) and 15(a)] and layer development [Figs. 14(b) and 15(b)] are consistent with the experimental results. The characteristics of the flow field are also in good agreement with that discussed previously. The convection in the cells and in a layered structure is more vigorous at positive angles than that at the negative ones for fixed $|\gamma|$. For $\gamma>0^{\circ}$, the fluid would flow in the entire cavity, but only in part of the cavity for $\gamma<0^{\circ}$. A minor discrepancy in respect to time exists. This may be attributed to the three-dimensional effect in the experiments and the idealization in the numerical simulation.

\section{CONCLUSION}

The inclined angle influences the onset of instability, layer number, layer structure and the layer thickness. For a fixed $|\gamma|$, the onset of instability at positive angles is slower than that at the negative ones. The phenomenon of asymmetry, which results from the different effects of vertical solutal gradient prior to the onset of instability, is the same as the results in a narrow slot [17-19].

More vigorous convection occurs at the positive angles than the negative ones. This is due to the heating-from-below effect. At $y=0^{\circ}$ the cell numbers are the greatest. At positive angles there are more cells than at negative angles for the same $|\gamma|$. However, the layer numbers have opposite tendency due to the more rapid merging at positive angles. The aspect ratio and the orientation also have a marked effect on the flow type, especially in the top and bottom layers. The convective flow will occupy the whole domain at positive angles, but only part of the domain at negative angles. The time needed to get to the steady state is maximum as $\gamma=0^{\circ}$ and reduces as the angle increases or decreases. The temperature and solutal distributions reveal the heat and mass transfer behaviors of the layered structure.

From the local Nusselt number profiles, the distributions are wavy due to the layered structure, and the inclined angle has a profound effect on them. Merging is a dissipation process, and so, the average Nusselt number reduces; while the forming of a new structure is a restoration process and the average Nusselt number involves an abrupt rising. At positive angles the plots vibrate strikingly, while these are smoother at negative angles.

The other kind of flow type occurs as the cavity inclines nearly horizontally, e.g. at $\gamma \geqslant 75^{\circ}$. Some pairs of vortexes form a well-mixed fluid layer. This kind of onset of instability is similar to the case in the horizontal fluid layer at $\gamma=90^{\circ}$, for which the temperature and solutal distribution are uniform, and differs from the cases described previously.

The comparison of the final layer thickness in our results and the existing literatures verifies the accuracy 


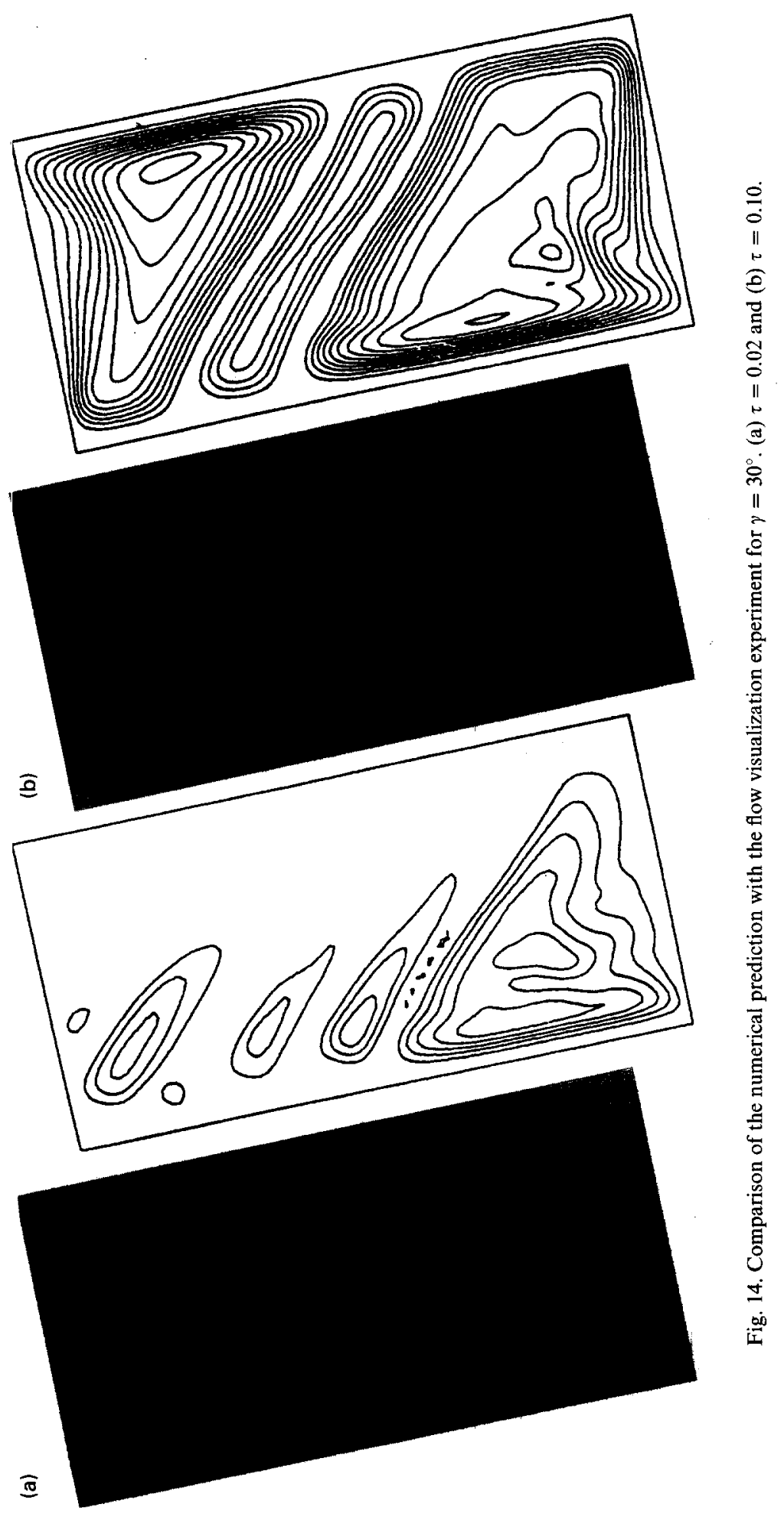




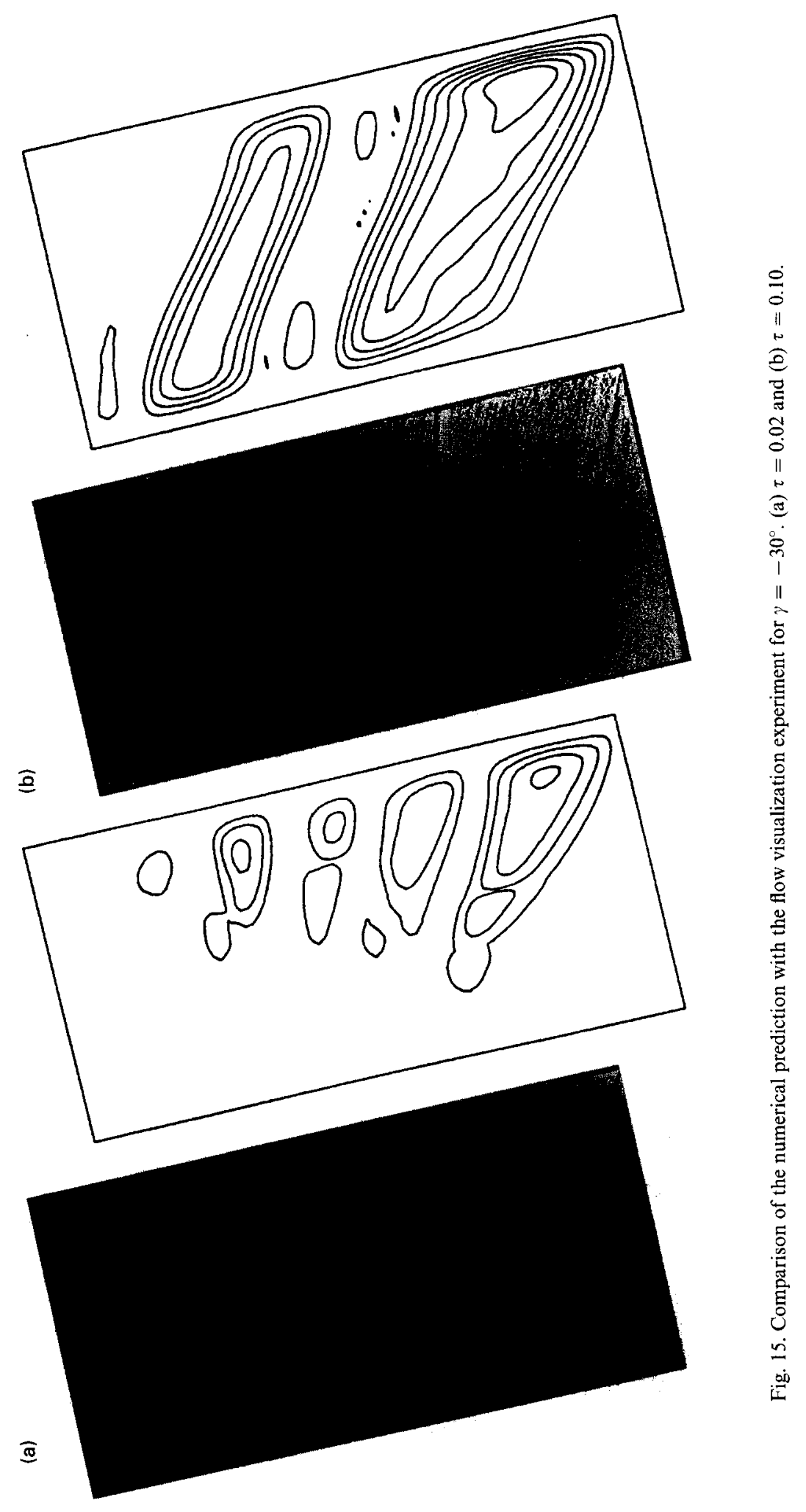


of our numerical predictions. The final layer thicknesses are thicker at positive angles than that at negative angles owing to the vigorous merging process.

\section{REFERENCES}

1. J. S. Turner, Multi-component convection, A. Rev. Fluid Mech. 17, 11-44 (1985)

2. S. Ostrach, Natural convection with combined driving forces, Physico Chem. Hydrodyn. 1, 233-247 (1980).

3. J.S. Turner, Buovancy Effects in Fluids. Cambridge University Press, Cambridge (1973).

4. S. A. Thorpe, P. K. Hutt and R. Soulsby, The effect of horizontal gradients on thermohaline convection, $J$. Fluid Mech. 38, 375-400 (1969).

5. C. F. Chen, D. G. Briggs and R. A. Writz, Stability of thermal convection in a salinity gradient due to lateral heating, Int. J. Heat Mass Transfer 14, 57-65 (1971).

6. H. E. Huppert and J. S. Turner, Ice blocks melting into a salinity gradient, J. Fluid Mech. 100, 367-384 (1980).

7. H. E. Huppert, R. C. Kerr and M. A. Halworth, Heating or cooling a stable compositional gradient from the side, Int. J. Heat Mas: Transfer 27, 1395-1401 (1984).

8. J. Tanny and A. B. Tsinober, The dynamics and structure of double-di:fusive layers in sidewall-heating experiments, J. Fluid Mech. 196, 135-156 (1988).

9. C. G. Jeevaraj and J. Imberger, Experimental study of double-diffusive instability in sidewall heating, $J$. Fluid Mech. 222, 565-586 (1991).

10. J. W. Lee and J. M. Hyun, Time-dependent double diffusion in a stably stratified fluid under lateral heating, Int. J. Heat Mass Transfer 34, 2409-2421 (1991).

11. K. Kamakura and H. Ozoe, Experimental and numerical analysis of double diffusive natural convection heated and cooled from opposed vertical walls with an initial condition of a vertically linear concentration gradient, Int. J. Heat Mass Transfer 36, 2125-2134 (1993).

12. J. Dosch and H. Beer, Numerical simulation and holographic visualization of double diffusive convection in a horizontal concentric annulus, Int. J. Heat Mass Transfer 35, 1811--1821 (1992).
13. C. F. Chen, Time-dependent double-diffusive instability in a density-stratified fluid along a heated inclined wall, ASME J. Heat Transfer 100, 653-658 (1978).

14. M. Kazmierczak and D. Poulikakos, Transient double diffusion in a layer heated from below, Int. J. Heat Fluid Flow 11, 30-39 (1990).

15. W. T. Lewis, F. P. Incropera and R. Viskanta, Interferometric study of stable salinity gradients heated from below or cooled from above, J. Fluid Mech. 116, 411$430(1982)$

16. C. F. Chen, R. C. Paliwal and S. B. Wong, Cellular convection in a density stratified fluid: effect of inclination of the heated wall, Proceedings of the 1976 Heat Transfer and Fluid Mechanics Institute, Vol. 78, pp. 1832 (1976).

17. R. C. Paliwal and C. F. Chen, Double-diffusive instability in an inclined fluid layer. Part 1. Experimental investigation, J. Fluid Mech. 98, 755-768 (1980).

18. R. C. Paliwal and C. F. Chen, Double-diffusive instability in an inclined fluid layer. Part 2. Stability analysis, J. Fluid Mech. 98, 769-785 (1980).

19. S. Thangam, A. Zebib and C. F. Chen, Double-diffusive convection in an inclined fluid layer, J. Fluid Mech. 116, 303-378 (1982).

20. O. M. Phillips, On flows induced in a stably stratified fluid, Deep-Sea Res. 17, 435-443 (1970).

21. C. E. Mendenhall and M. Mason, The stratified subsidence of fine particles, Proc. US Natl Acad. Sci. 9, 199202 (1923)

22. S. L. Lee, Weighting function scheme and its application on multidimensional conservation equations, Int. J. Heat Mass Transfer 32, 2065-2073 (1989).

23. S. L. Lee and R. Y. Tzong, Artificial pressure for pressure linked equation, Int. J. Heat Mass Transfer. 35, 2705-2716 (1992)

24. D. G. Neilson and F. P. Incropera, Double-diffusive flow and heat transfer for a cylindrical source submerged in a cylindrical source submerged in a salty-stratified solution, Int. J. Heat Mass Transfer 30, 2559-2570 (1987).

25. T. L. Bergman and A. Ungan, A note on lateral heating in a double diffusive system, $J$. Fluid Mech. 194, 175-186 (1988). 